\title{
Focusing wave group on a current of finite depth
}

\author{
D. Merkoune ${ }^{1}$, J. Touboul ${ }^{2}$, N. Abcha ${ }^{1}$, D. Mouazé ${ }^{1}$, and A. Ezersky ${ }^{1}$ \\ ${ }^{1}$ Laboratoire de Morphodynamique Continental et Côtier, UMR6143, CNRS, 24 rue des Tilleuls, 14000 Caen, France \\ ${ }^{2}$ Université de Toulon, CNRS/INSU, IRD, MIO, UM 110, 83957 La Garde, France
}

Correspondence to: A. Ezersky (alexander.ezersky@unicaen.fr)

Received: 10 December 2012 - Published in Nat. Hazards Earth Syst. Sci. Discuss.: -

Revised: 12 October 2013 - Accepted: 16 October 2013 - Published: 19 November 2013

\begin{abstract}
Formation of freak waves resulting from the wave packets propagating in finite water depth on the background of a current is studied experimentally and numerically. In the experiment, the freak waves appear as a result of dispersion focusing of wave train excited by wave maker with modulated frequency. The space evolution of the frequency modulated train is studied in numerical simulations. We showed that in the water of finite depth, a distance of focusing increases and amplitude in the focal point decreases in comparison with infinite water depth. Experimental results are in good agreement with numerical simulations if wave breaking of surface waves does not occur.
\end{abstract}

\section{Introduction}

The waves of large amplitudes, called freak waves, suddenly appearing in the ocean and have a complex origin that is a big challenge to maritime safety. Their appearance is observed during storms of certain intensity, usually in the open ocean in deep water (Lawton, 2001). These waves are due to a large variety of physical mechanisms, explaining the large number of locations where rogue waves were observed (Kharif and Pelinovsky, 2003). Among others, one may cite wave current interaction. Indeed, several accidents were reported in the Aguhlas Current of South Africa (Mallory, 1974). These accidents were explained through the wave current interaction, often leading to a spatial focusing of water waves (Lavrenov, 1998; White and Fornberg, 1998).

The modulational instability, also known as BenjaminFeir instability (Benjamin and Feir, 1967), is often cited in the framework of freak wave formation (Yuen and Lake, 1980). It has been widely used to study rogue wave statistics, and its deviation from linear theory (Onorato et al., 2001). More recently, the influence of wind (Touboul and Kharif,
2006), or current (Onorato et al., 2011; Toffoli et al., 2011, 2013) on the dynamics of modulation instability was also investigated.

Dispersive focusing, which corresponds to the spatiotemporal focusing of frequency modulated wave trains, is also known to play a significant role in the formation of rogue waves (Baldock et al., 1996; Kharif et al., 2001; Porubov et al., 2005). The compression of a wave train with modulated frequency is due to wave dispersion. Since long waves propagate faster than short ones, when long waves are situated at the tail of a wave train, they will overtake short waves initially located ahead the wave group. Touboul et al. (2006) showed experimentally and numerically that wind might significantly increase the height and lifetime of rogue waves due to dispersive focusing. Chambarel et al. (2010a, b) confirmed the result for focusing wave groups in shallow water in the presence of wind.

Recently, Touboul et al. (2007) studied theoretically the influence of a current on the focusing of wave groups propagating in deep water. They concluded that the current had a defocusing effect, leading to a decrease of the freak wave heights, which was compensated in opposing current by nonlinear effects.

However, it is clear that, in coastal zone the deep water approximation is not valid anymore. It is necessary to take into account the bathymetry effects, which play an important role in the dispersive nature of the wave train. Paradoxically, the focusing of wave train in these conditions has never been studied. In this work, we focus our attention on study of finite depth effects. We investigated experimentally and numerically the propagation of a wave train with the modulated frequency with and without current. Linear and non-linear effects of a current on focusing wave characteristics are discussed in details. 


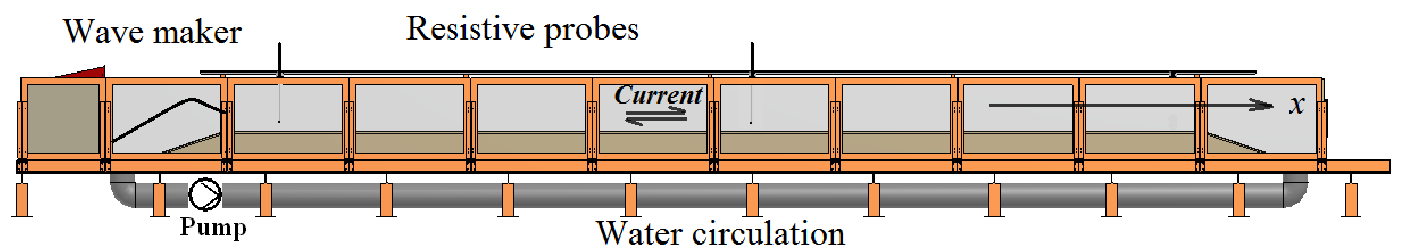

Fig. 1. Experimental set-up.

\section{Experimental set-up}

The experimental study is conducted in the wave flume of the Laboratory of Continental and Coastal Morphodynamics, in Caen. This flume (Fig. 1) has a length of $18 \mathrm{~m}$, a width of $0.5 \mathrm{~m}$, while water depth varies from 0 to $0.4 \mathrm{~m}$. Waves of different frequencies and amplitudes are generated in the flume under conditions of deep water and shallow water. To generate surface waves in the flume, a computer controlled wavemaker is used. Owing to numerically synthesized signals, it is possible to excite wave train with modulated frequency. Experimental facilities allow us to investigate propagation of surface wave on the background of a steady current. This current is generated by means of power pump, as indicated on Fig. 1. To decrease disturbances created by the pump and for the purpose of reducing wave reflection, a special honeycomb is installed at the end wall of the tank, opposite to the wave-maker. Working regimes of the pump allows us to change flow rate values and the direction of current in the channel. Therefore two cases have been investigated: in the first case surface waves and current are co-directed, while in the second case, they are counter directed. To study the evolution of free surface displacement along the channel resistive probes are used. In the meantime, the characteristics of the steady current are obtained thanks to ultrasonic Doppler velocimetry (USDV); USDV provides us the instantaneous velocity profiles.

To understand the influence of finite depth effects, packets with different frequencies are excited: a low frequency packet, with a spectral peak of $f_{\max }=0.75 \mathrm{~Hz}$ corresponds to carbonate hardness $(\mathrm{kh})=0.9$ in the absence of current; a intermediate frequency packet of $f_{\max }=1.2 \mathrm{~Hz} \mathrm{kh}=1.8$; and high frequency packet of $f_{\max }=1.7 \mathrm{~Hz}, \mathrm{kh}=2.95$. The last case corresponds to deep water conditions.

\section{Experimental results}

\subsection{Evolution of wave packet energy}

In the experiments, the focusing of wave packets occurs on the background of wave energy dissipation. For this reason, and before investigating the focusing effects due to frequency modulation, we study the energy of wave packet propagating in the flume. We compare wave packet energy near the

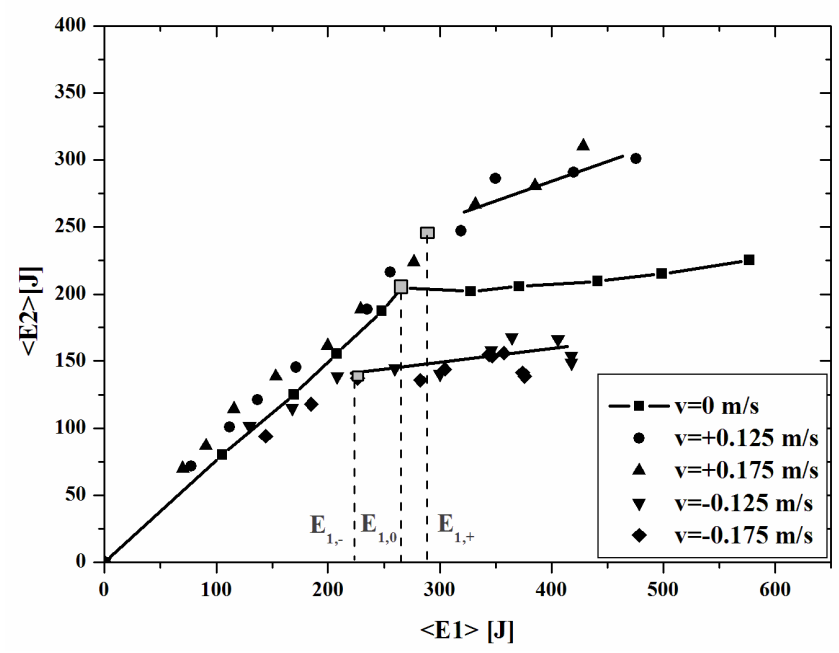

Fig. 2. Dependence of low frequency $\left(f_{\max }=0.75\right)$ wave packet energy $E_{2}$ (energy measured at $x=15 \mathrm{~m}$ ) on $E_{1}$ (energy measured at $x=1 \mathrm{~m})$.

wave maker with wave packet energy at a large distance. The wave packet energy (energy on a unit length in the direction transversal in the direction of wave propagation) is estimated as follows:

$E=\frac{\rho g}{2} c_{\mathrm{gr}} \int(\eta-<\eta>)^{2} \mathrm{~d} t$,

where $C_{\mathrm{gr}}=\frac{\mathrm{d} \omega}{\mathrm{d} k}$ is the group velocity of harmonic component corresponding to the peak frequency $f_{\max }, g$ for acceleration of gravity, $\rho$ water density, $T$ duration of impulse, $\eta$ and $\langle\eta\rangle$ are free surface displacement and mean water level, respectively.

Typical dependences of $E_{2}$ (energy at $x=15 \mathrm{~m}$ ) on $E_{1}$ (energy at $x=1 \mathrm{~m}$ ) are shown in Fig. 2 for different velocity of current. For small energy linear dependence $E_{2}\left(E_{1}\right)=$ $\beta E_{1}(\beta=0.75)$ is observed. Such linear dependence (decreasing of energy by $25 \%$ ) is not due to wave breaking. These losses are caused by viscous dissipation and contactline damping. It should be noted that in our experiments losses of energy are larger than in previously published papers (see for example Tian et al., 2010). This difference can be explained by the fact that in present experiments the influence of viscose boundary layers at sidewalls and bottom 
is larger than in Tian et al. (2010) because measurements are performed at smaller flume width and water depth. Theoretical estimations show that if take into consideration molecular viscosity of water and boundary layers at walls and bottom, energy decay at $15 \mathrm{~m}$ is $18 \%$. Note that in the experiment there exist additional effects leading to the energy loses: non-controlled roughness of walls, dissipation in boundary layer at free surface, in the bulk of the water and contact-line damping.

For large amplitude of the wave packet, spilling breaker waves appears and dependence became more important (a high energy dissipation). Wave breaking occurs if $E_{1}>E_{1,0}$ in the absence of current, for $E_{1}>E_{1,+}$, if the direction of flow coincides with the wave packet direction of propagation, and $E_{1}>E_{1,-}$, if directions of flow and packet propagation are opposite. We investigate evolution of wave packets for the case of linear energy dissipation, influence of dissipation of the energy due to wave breaking may be is very small under our experimental conditions.

\subsection{Evolution of wave packet}

Three series of measurements for frequencies of wave packet $f_{\max }=0.75 \mathrm{~Hz}, f_{\max }=1.2 \mathrm{~Hz}$ and $f_{\max }=1.7 \mathrm{~Hz}$ have been carried out. In the first and third cases water depth was $h=0.25 \mathrm{~m}(\mathrm{kh}=0.9$ and $\mathrm{kh}=2.95)$ for the absence of current and in the second one $h=0.32 \mathrm{~m}(\mathrm{kh}=1.8)$. For these cases the influence of current has been studied in details. First of all profiles of current velocity were investigated. They are presented in Fig. 3. Characteristic profile of mean velocity and root mean square fluctuations of velocity at a distance $3 \mathrm{~m}$ from the wave maker are shown in Fig. 3. Velocity has boundary layer near the bottom, thickness was approximately $3 \mathrm{~cm}$. In the a boundary layer, near the bottom, velocity fluctuations are sufficiently larger than near the free surface. Such mean velocity and fluctuations were observed for positive and negative values of water currents far from wave maker. Meanwhile near the wave maker, velocity profiles for negative and positive current flow were different (Fig. 4a and b). For positive currents a classic velocity profile with boundary layer of several centimetres was observed. Profiles of negative velocities have maximum velocity near the bottom. It is due to our experimental installation. When negative flow is created, water is sucked in by pump near the wave maker. When positive flow is created water is injected at the opposite end of our flume, $17 \mathrm{~m}$ from the channel bottom near the wave-maker.

Wave train generated by wave maker propagates along the flume and its shape changes during the propagation (see Fig. 5, dotted line). This process can be described by two main parameters: by maximal wave height appearing as a result of dispersion focusing and by the distance at which this maximal amplitude is reached. The dependences of these parameters on flow velocity in the channel is shown in Figs. 6 and 7. It was found that wave train that propagates on the

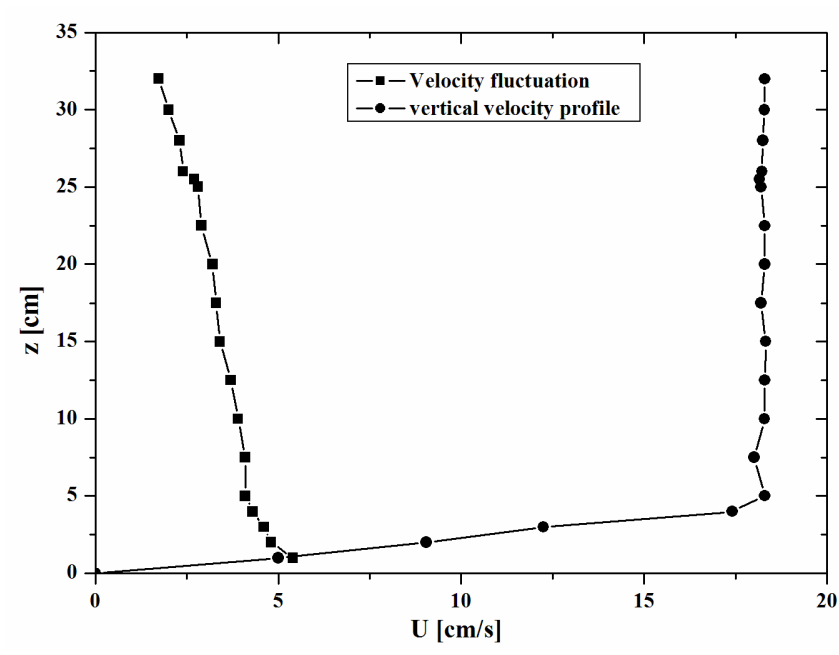

Fig. 3. Profiles of current velocity: mean profile and mean root velocity fluctuations at $3 \mathrm{~m}$ from the wave maker.

background of co-directed current focuses at a greater distance from the wave maker than wave train propagating on the background of counter directed current. This effect is most pronounced in the case of low frequency wave train $(\mathrm{kh}=0.9)$ than for others wave trains. The increasing of wave height in our experiments in some cases can lead to large local steepness that is a bit less than critical value above which wave breaking at the focusing point appears. In this sense, the results presented in Figs. 6 and 7 are in agreement with the experiments of $\mathrm{Wu}$ and Yao (2004) aimed to investigate the position of wave breaking point in the presence of a current. They founded that co-directed current slows down the wave breaking and counter-directed current accelerates the wave breaking. In our experiments wave breaking is not observed but a shift of the point corresponding to maximal amplitude is the same as the shift of the wave braking point in Wu and Yao (2004). Dependences of maximum elevation of the free surface at the focusing point versus the current velocity for three cases of excitation frequency $\left(f_{\max }=0.75 \mathrm{~Hz}\right.$, $f_{\max }=1.2 \mathrm{~Hz}$ and $\left.f_{\max }=1.7 \mathrm{~Hz}\right)$ are presented in Fig. 6 in non-dimensional form. In Fig. $6, A_{\max } / A_{0 \max }$ is the ratio of the maximum wave height at the focusing point and the maximum wave height of the wave train near the wave maker, $U / C_{\mathrm{g}}$ is the ratio of the flow velocity and the group velocity of harmonic corresponding the spectral maximum (peak frequency). It was found that in the absence of current, the amplification factor is up to $40 \%$ for both waves in water of finite depth, where as in deep water this factor may be close to $70 \%$. For waves propagating in water of finite depth, the amplification factors practically coincide for small velocity of current $\left|U / C_{\mathrm{g}}\right|<0.05$. For larger current velocities, the amplification factors differ sufficiently and depend on correlation between wave length and water depth. 

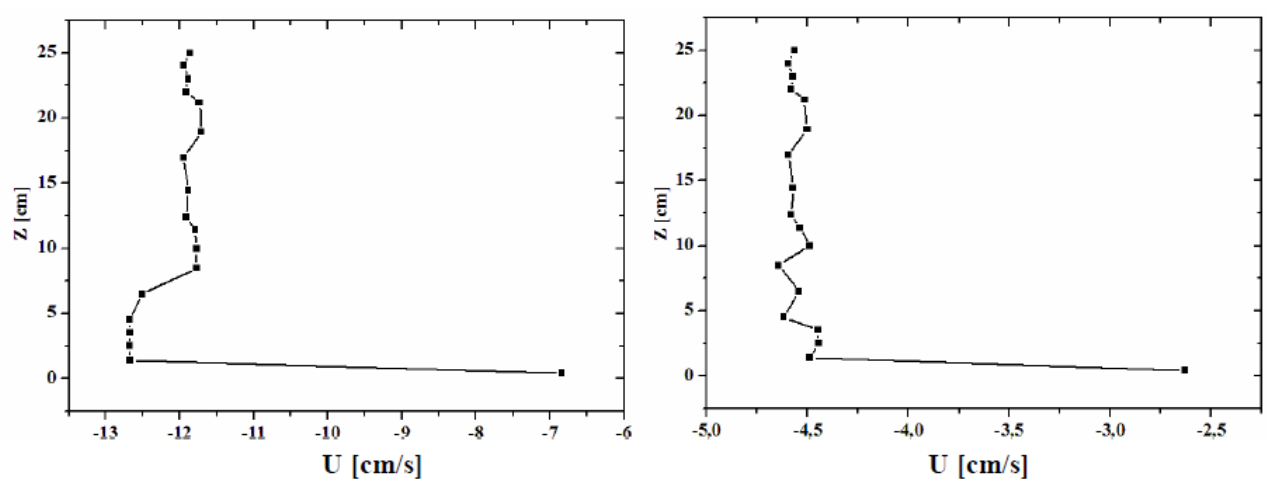

(a)
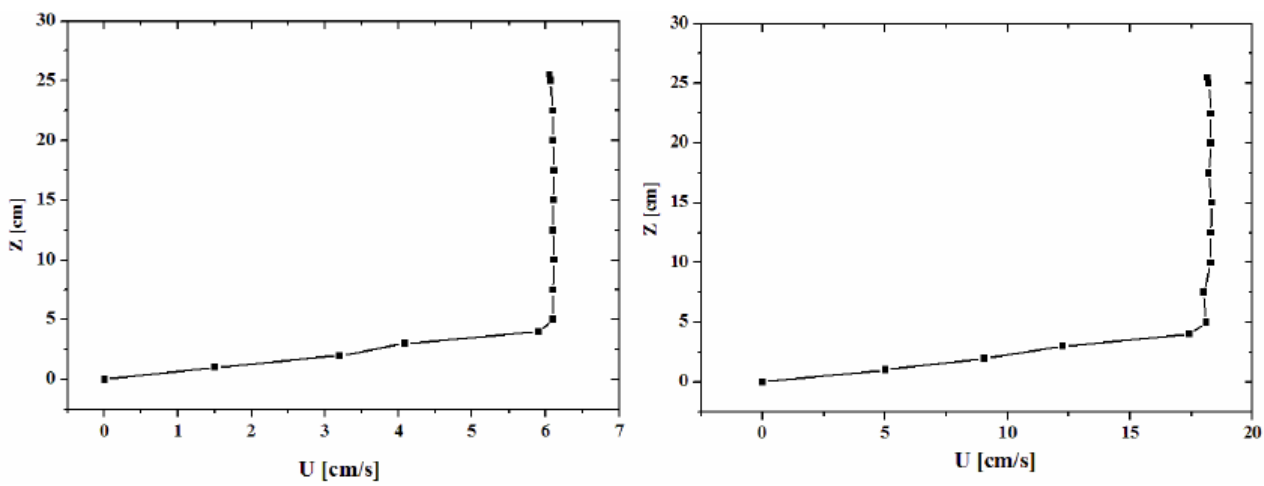

(b)

Fig. 4. (a) profiles of velocity for negative current in the vicinity of wave maker. (b) profiles of velocity for positive current in the vicinity of wave maker.
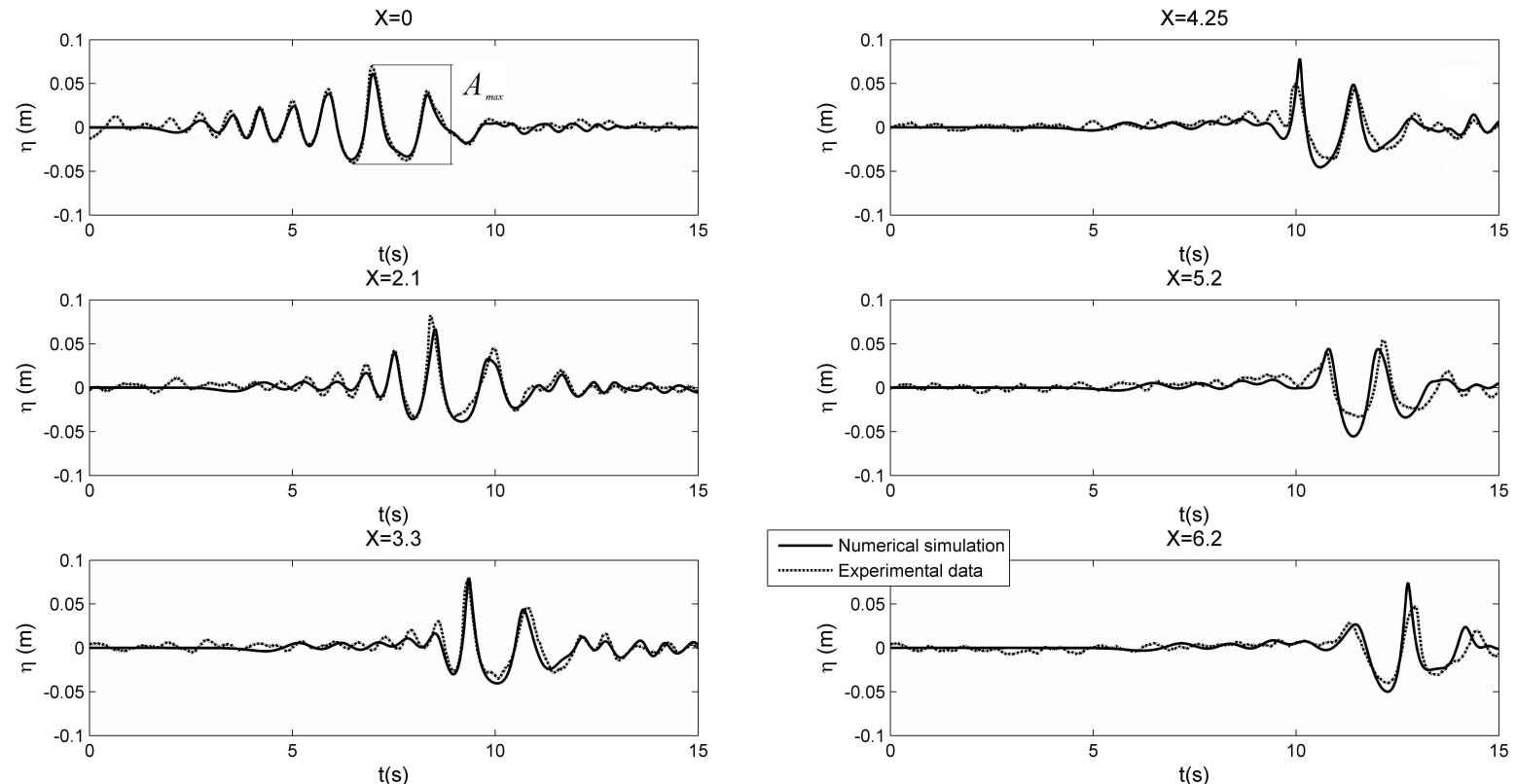

Fig. 5. Space-time evolution of impulse generated by wave maker. Dotted curves correspond to experimental data, solid curves are results of numerical simulations. 


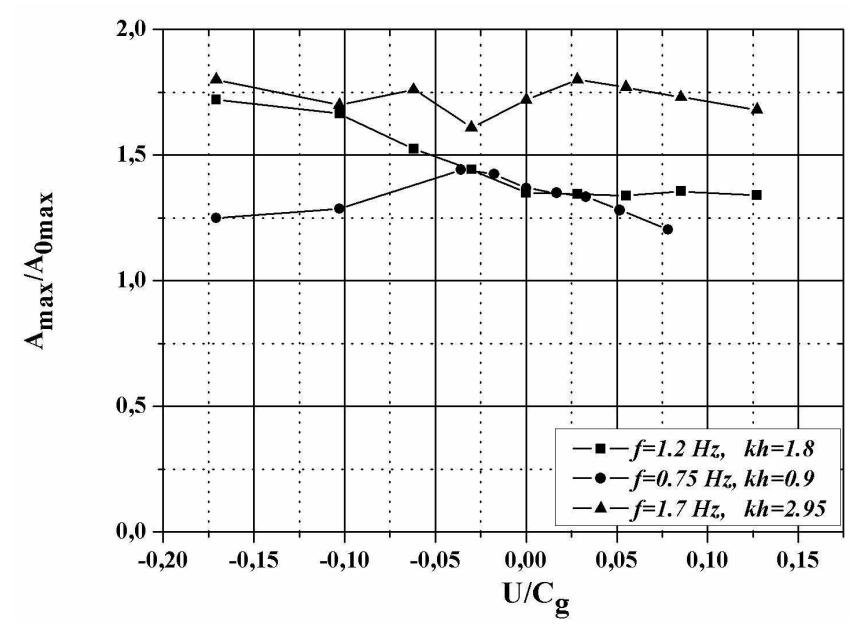

Fig. 6. Dependence of the amplitude in the focusing point on velocity of hydrodynamic flow for frequency modulated wave train: $f_{\max }=0.75 \mathrm{~Hz}, f_{\max }=1.2 \mathrm{~Hz}$ and $f_{\max }=1.7 \mathrm{~Hz}$.

Dependencies of focusing point positions on current velocity are shown in Fig. 7. It is possible to conclude that focusing distance decreases with increasing of parameter kh: wave packet in deep water has more amplification factor than in a water layer of finite depth, especially for the case of positive current, when direction of wave propagation and direction of hydrodynamic current coincide.

\subsection{Spectral analysis}

In order to investigate the influence of finite water depth on interaction of frequency modulated wave train with hydrodynamic flow, the spectral analyses of free surface elevations were performed for two frequencies: $f_{\max }=1.2 \mathrm{~Hz}$ and $f_{\max }=0.75 \mathrm{~Hz}$. The initial spectra of free surface elevation (spectra measured in the vicinity of wave maker) and spectra measured at $x=6 \mathrm{~m}$ are shown in Fig. 8a and b in the absence of current $U=0$, and for $U=0.12 \mathrm{~m} \mathrm{~s}^{-1}$, $U=-0.12 \mathrm{~m} \mathrm{~s}^{-1}$.

One can see that the shape of wave train spectrum with peak frequency $f_{\max }=1.2 \mathrm{~Hz}$ differs from the shape of lower frequency wave train spectrum with $f_{\max }=0.75 \mathrm{~Hz}$. In the second spectra it is possible to find harmonics of the peak frequency $f_{\max }=0.75 \mathrm{~Hz}$. They are indicated in Fig. $8 \mathrm{~b}$ by arrows. Harmonics multiplied to the $f_{\max }$ have smaller amplitudes for the high frequency wave train. The generation of harmonics is due to non-linearity of surface waves. Such generation is more effective in the case of small dispersion (low frequency waves train with $f_{\max }=0.75 \mathrm{~Hz}$ ). The comparison of spectra presented in Fig. 8a and b shows that different spectral components demonstrate different behaviour on the background of hydrodynamic flow. For wave trains propagating upstream, high frequency components decay more than in the wave train propagating downstream

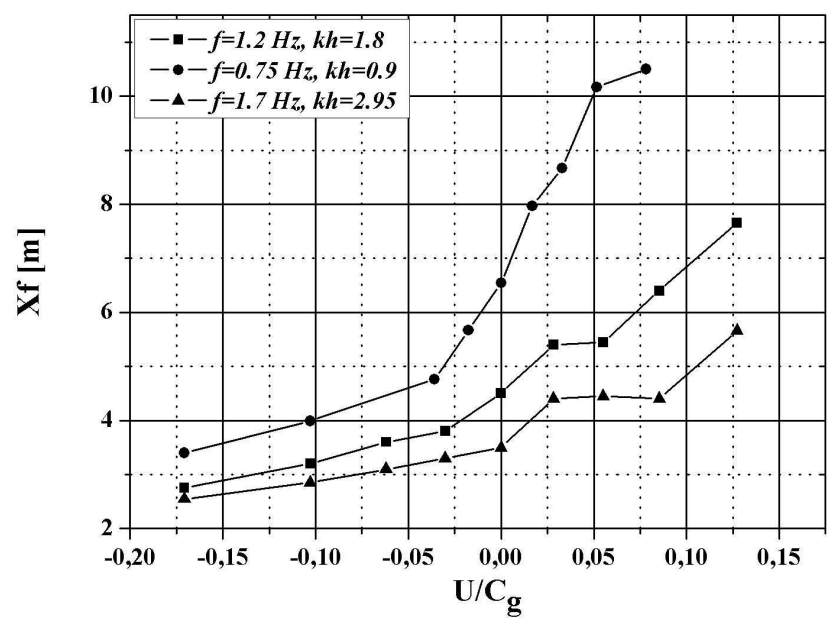

Fig. 7. Dependence of focusing point position on velocity of hydrodynamic flow for frequency modulated wave train: $f_{\max }=0.75 \mathrm{~Hz}$, $f_{\max }=1.2 \mathrm{~Hz}$ and $f_{\max }=1.7 \mathrm{~Hz}$.

(compare for example Fig. 8a II and Fig. 8a III; Fig. 8b II and Fig. $8 \mathrm{~b}$ II). It should be noted that the thickness of the main spectral peak does increase with the distance from the wave maker. It is observed for spectra presented in Fig. 8. Therefore, in our experiments modulation instability leading to the broadening spectral peak does not occur. Such result seems natural for our experimental conditions. For wave train with peak frequency $f_{\max }=0.75 \mathrm{~Hz}(\mathrm{kh}=0.9)$ conditions for modulation instability are not satisfied. It is known (Whitham, 1974) that modulation instability occurs if $\mathrm{kh}>1.3$. For other wave trains conditions for modulation, instabilities are satisfied but it is evidently that the distance of wave train propagation is not sufficient for the development of this kind of instability.

\section{Numerical simulation and comparison with experiment}

For numerical simulations potential theory is used. By assuming the fluid to be inviscid, incompressible, animated with an irrotational motion, it is known that the fluid velocity derives from a velocity potential $\varphi(x, z, t)$, which satisfies the Laplace equation $\Delta \varphi=0$. Thus, this velocity potential might be related to the water elevation $\eta(x, t)$ by means of the nonlinear boundary conditions of the problem. Indeed, the problem is complete while the bottom and free surface boundary conditions are considered together with the Laplace equation: 

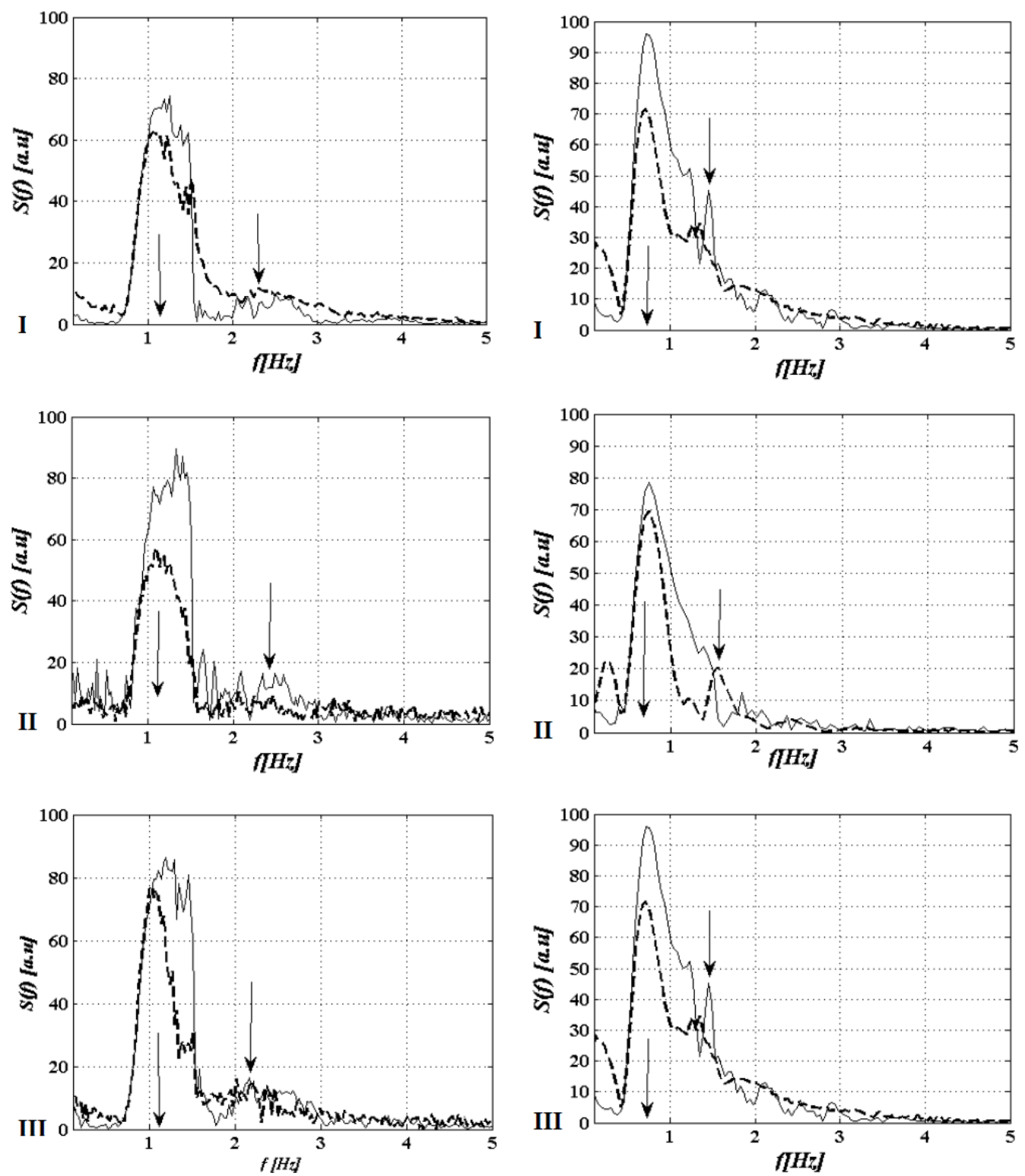

(a)

(b)

Fig. 8. (a) Comparison of spectra $S(f)$ (arbitrary units) at distance $x=0$ (solid curve) and $x=6 \mathrm{~cm}$ (dotted curve for $f_{\text {max }}=1.2 \mathrm{~Hz}$ : (I) $U=0$, (II) $U=0.12 \mathrm{~m} \mathrm{~s}^{-1}$, (III) $U=-0.12 \mathrm{~m} \mathrm{~s}^{-1}$ ). (b) Comparison of spectra $S(f)$ (arbitrary units) at distance $x=0$ (solid curve) and $x=6 \mathrm{~cm}$ (dotted curve for $f_{\max }=0.75 \mathrm{~Hz}$ : (I) $U=0$, (II) $U=0.12 \mathrm{~m} \mathrm{~s}^{-1}$, (III) $U=-0.12 \mathrm{~m} \mathrm{~s}^{-1}$ ).

$$
\begin{aligned}
& \phi=\varphi+U x \\
& \Delta \varphi=0 \\
& \frac{\partial \eta}{\partial t}+\frac{\partial \varphi}{\partial x} \frac{\partial \eta}{\partial x}+U \frac{\partial \eta}{\partial x}=\frac{\partial \varphi}{\partial z} \\
& \text { and } \frac{\partial \varphi}{\partial t}+U \frac{\partial \varphi}{\partial x}+\frac{(\nabla \varphi)^{2}}{2}+g \eta=0 \\
& \text { on } z=\eta(x, t) \\
& \frac{\partial \varphi}{\partial z}=0 \text { on } z=-h .
\end{aligned}
$$

If linearized, the kinematic and dynamic boundary conditions admit harmonic waves for solution. In this case, the linear dispersion relation

$(\omega-k U)^{2}=g k \tanh (k h)$

must be satisfied. Thus, components obtained by means of Fourier transform of an appropriate initial boundary condition might be advected independently. An inverse Fourier transform would then provide the time evolution of the water elevation 
$\eta(x, t)=\frac{1}{2 \pi} \int\left(\int \eta(0, \tau) e^{i \omega t} \mathrm{~d} \tau\right) e^{i(k x-\omega t)} \mathrm{d} \omega$.

To solve the fully nonlinear problem for $\varphi$, described by Eqs. (3)-(5) a mixed Euler-Lagrange description of the problem is adopted, meaning that a particular description of the surface is used. More details can be found in Touboul et al. (2006). This method has been checked by comparison with numerical simulations by Zhu and Zhang (1997), and a good agreement has been found. It should be emphasized that these approaches do not take into account the interaction of wave train with turbulence of hydrodynamic flows.

Figure 5 shows the comparison of experimental (dotted curve) and numerical results (solid curve) for the case of zero velocity $U=0$. Experimentally, measured time dependence of free surface displacement at $x=0$ is used as a boundary condition in numerical simulations. The comparison of experimental data and numerical results are shown at different distances along the channel; thus, it displays good correlation between free surface displacement in experiments and in numerical simulations. Some calculations were performed to compare maximal wave height amplification and location of the focusing point measured in the channel. Figure 9 presents comparison of focusing point position. We have found good agreement between experimental and numerical results. Figure 10 shows the comparison of maximum amplification amplitude reached by the waves as a function of current velocity for water of finite depth and deep water. Good agreement is observed for positive velocity of the current. For negative velocity differences between numerical and experimental data may reach $50 \%$ (see Fig. 9b). The main reason for this difference is the profile of shear flow near the wave maker (Fig. 4b). Profile of the velocity has maximum its near the bottom. Shear stresses are not taken into consideration in the numerical model.

\section{Conclusions}

We found that the focusing distance depends on the direction of hydrodynamic flow. This length increases for waves propagating in the direction of flow and decreases for the opposite direction. We also showed that this length decreases with decreasing of ratio $h / \lambda$ (where $\lambda$ is the wavelength). Naturally this result is due to the decreasing of surface waves dispersion. Dispersion is also responsible for the different maximum wave height at the focusing point. In the case of finite depth of water, the elevation of the amplitude in the presence of a current is smaller compared with the case of deep water. The numerical simulations were performed for the equations of inviscid fluid for wave propagating on the background of homogeneous flow. It was found that the experimental results are in good agreement with numerical simulations, if non-dimensional current velocity is not large and the breaking of surface waves does not occur during their "focusing".

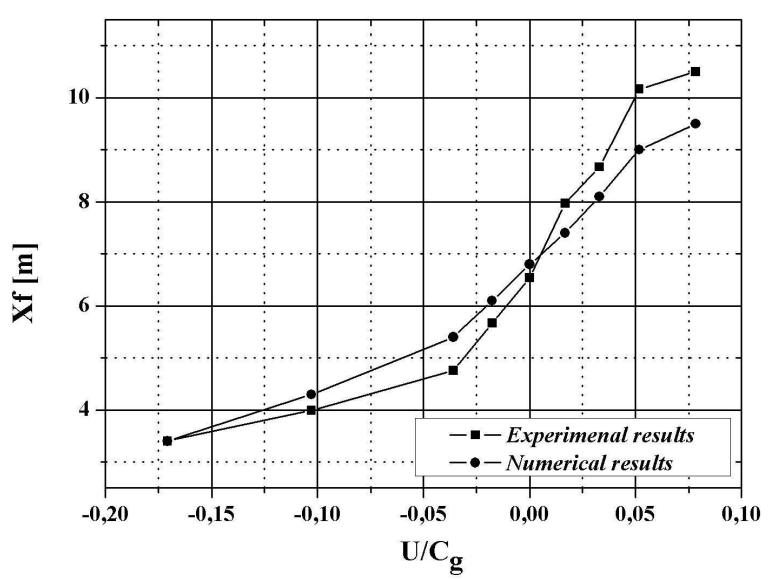

(a)

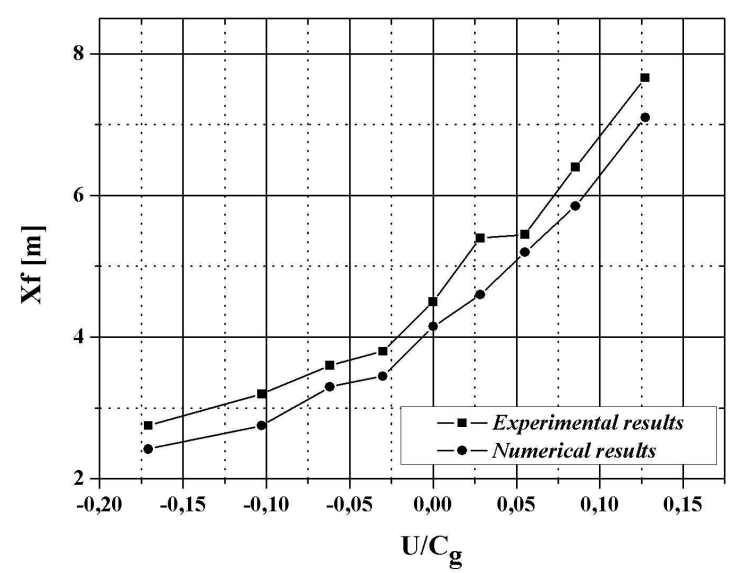

(b)

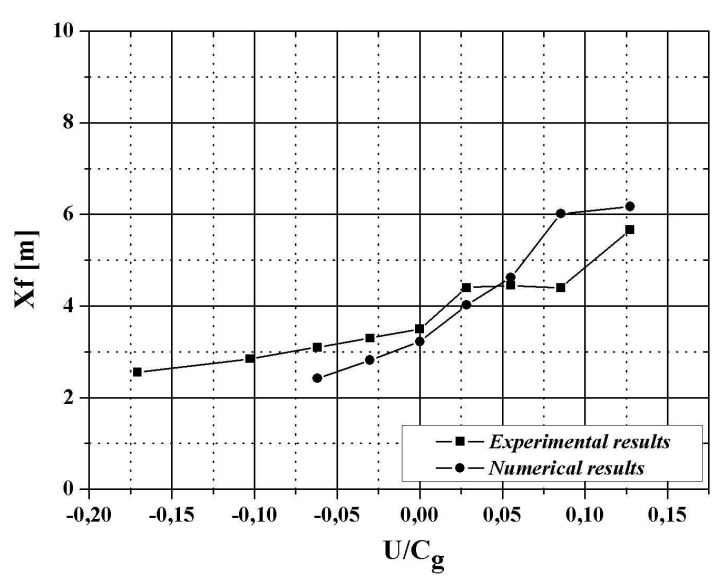

(c)

Fig. 9. Comparison of experimental and theoretical results on focusing point for wave train with (a) $f_{\max }=0.75 \mathrm{~Hz}$, (b) $f_{\max }=$ $1.2 \mathrm{~Hz}$ and (c) $f_{\max }=1.7 \mathrm{~Hz}$. 


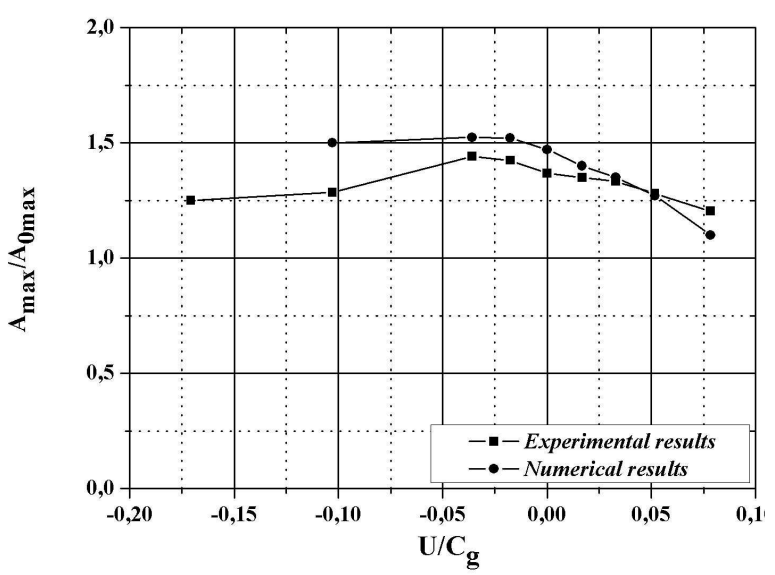

(a)

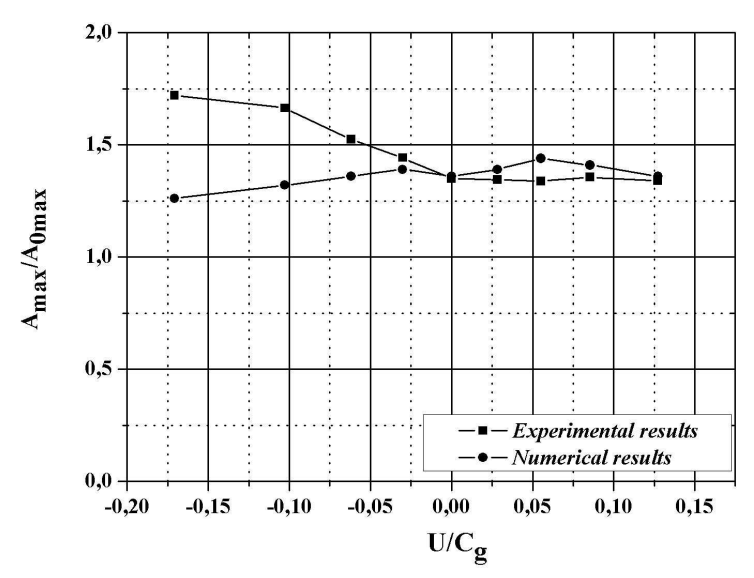

(b)

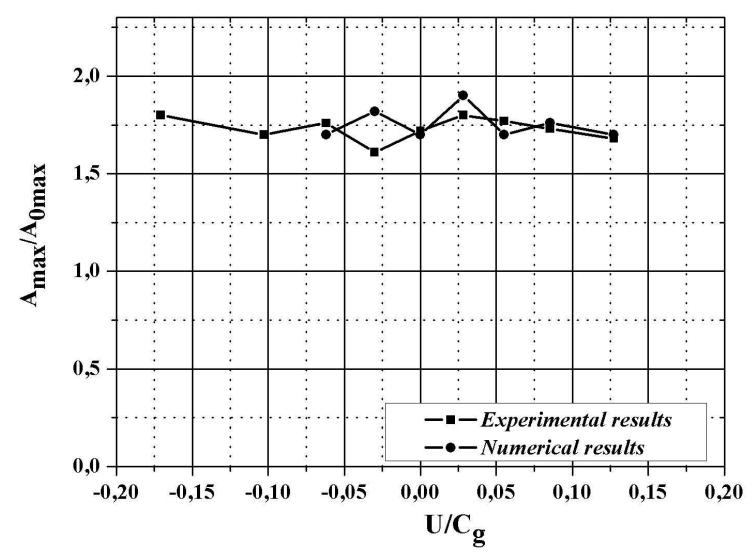

(c)

Fig. 10. Comparison of experimental and numerical results on amplitude in the focusing point for wave train (a) $f_{\max }=0.75 \mathrm{~Hz}$, (b) $f_{\max }=1.2 \mathrm{~Hz}$ and (c) $f_{\max }=1.7 \mathrm{~Hz}$.
For a more precise comparison of experimental and numerical results, it is necessary to take into consideration nonpotentiality of the velocity field due to the vertical shear of hydrodynamic current.

Acknowledgements. We thank the reviewers for productive criticism.

Edited by: A. Toffoli

Reviewed by: O. Kimmoun and two anonymous referees

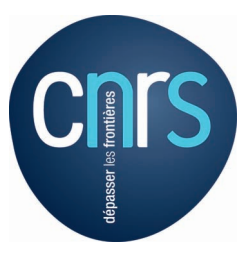

The publication of this article is financed by CNRS-INSU.

\section{References}

Baldock, T. E., Swan, C., and Taylor, P. H.: A laboratory study of surface waves on water, Phil Trans. R. Soc. Lond. A, 354, 649676, 1996.

Benjamin, T. B. and Feir, J. E.: The disintegration of wave trains on deep water. Part 1: theory, J. Fluid Mech., 27, 417-430, 1967.

Chambarel, J., Kharif, C., and Kimmoun, C.: Generation of twodimensional steep water waves on finite depth with and without wind. Eur. J. Mech. B. Fluids, 29, 132-142, 2010a.

Chambarel, J., Kharif, C., and Kimmoun, O.: Focusing wave group in shallow water in the presence of wind, Discrete and Continuous Dynamical Systems - Series B (DCDS-B), 13, 773-782, 2010b.

Kharif, C. and Pelinovsky, E.: Physical mechanisms of the rogue wave phenomenon, European J. Mechanics B, 22, 603-635, 2003.

Kharif, C., Pelinovsky, E., Talipova, T., and Slunyaev, A.: Focusing of Nonlinear Wave Groups in Deep Water, JETP Letters, 73, 170-175, 2001.

Lavrenov, I. V.: The wave energy concentration at the aguhlas current of South-Africa, Nat. Hazards, 17, 117-127, 1998.

Lawton, G.: Monsters of the deep (the perfect wave), New Scientist, 170 28-32, 2001.

Mallory, J. K.: Abnormal waves in the south east Africa, Int. Hydrog. Rev., 51, 89-129, 1974.

Onorato, M., Osborne, A. M., Serio, M., and Bertone, S.: Freak waves in random oceanic sea states. Phys. Rev. Lett., 86, 58315834, 2001.

Onorato, M., Proment, D., and Toffoli, A.: Triggering rogue waves in opposing currents. Phys. Rev. Lett., 107, 184502, doi:10.1103/PhysRevLett.107.184502, 2011.

Porubov, A. V., Tsuji, H., Lavrenov, I. V., and Oikawa, M.: Formation of the rogue wave due to nonlinear two-dimensional waves interaction, Wave Motion, 42, 202-210, 2005.

Tian, Z., Perlin, M., and Choi, W. : Energy dissipation in twodimensional unsteady plunging breakers and eddy viscosity model, J. Fluid Mech., 655, 217-257, 2010.

Toffoli, A., Cavaleri, L., Babanin, A. V., Benoit, M., BitnerGregersen, E. M., Monbaliu, J., Onorato, M., Osborne, A. R., 
and Stansberg, C. T.: Occurrence of extreme waves in threedimensional mechanically generated wave fields propagating over an oblique current, Nat. Hazards Earth Syst. Sci., 11, 895903, doi:10.5194/nhess-11-895-2011, 2011.

Toffoli, A., Waseda, T., Houtani, H., Kinoshita, T., Collins, K., Proment, D., and Onorato, M.: Excitation of rogue waves in a variable medium: An experimental study on the interaction of water waves and currents, Phys. Rew. E, 87, 051201-4, doi:10.1103/PhysRevE.87.051201, 2013.

Touboul, J. and Kharif, C.: On the interaction of wind and extreme gravity waves due to modulational instability, Phys. Fluids, 18, 108103, doi:10.1063/1.2374845, 2006.

Touboul, J., Pelinovsky, E., and Kharif, C.: Nonlinear Focusing Wave Group on Current, Coast. Ocean Eng., 19, 222-227, 2007.
Touboul, J., Giovanangeli, J.-P., Kharif, C., and Pelinovsky, E.: Freak waves under the action of wind: experimetns and simulations, Eur. J. Mech. B. Fluids, 25, 662-676, 2006.

White, B. S. and Fornberg, B.: On the chance of freak waves at sea, J. Fluid Mech., 355, 113-138, 1998.

Whitham, G. B.: Linear and nonlinear waves, Wiley-Interscience, New York, 1974.

$\mathrm{Wu}, \mathrm{C}$. H. and Yao, A.: Laboratory measurements of limiting freak waves on currents, J. Geophys. Res., 109, C12002, doi:10.1029/2004JC002612, 2004.

Yuen, H. C. and Lake, B. M.: Instabilities of waves on deep water, Ann. Rev. Fluid Mech., 12, 303-334, 1980.

Zhu, S. and Zhang, Y.: On nonlinear transient free-surface flows over a bottom obstruction, Phys. Fluids, 9, 2598-2604, 1997. 